\title{
Membrane current series monitoring: essential reduction of data points to finite number of stable parameters
}

\author{
Raoul R. Nigmatullin ${ }^{1}$, Rashid A. Giniatullin ${ }^{2,3}$ and Andrei I. Skorinkin ${ }^{4,5,6 *}$ \\ ${ }^{1}$ Theoretical Physics Department, Institute of Physics, Kazan Federal University, Kazan, Russia \\ ${ }_{2}^{2}$ Department of Neurobiology, A.I. Virtanen Institute, University of Eastern Finland, Kuopio, Finland \\ ${ }^{3}$ Laboratory of Neurobiology, Department of Physiology, Kazan Federal University, Kazan, Russia \\ ${ }^{4}$ Department of Radioelectronics, Institute of Physics, Kazan Federal University, Kazan, Russia \\ ${ }^{5}$ Department of Biophysics of Synaptic Processes, Kazan Institute of Biochemistry and Biophysics Russian Academy of Sciences, Kazan, Russia \\ ${ }^{6}$ Department of Bioinformatics, Institute of Informatics, Kazan, Russia
}

\section{Edited by:}

Tobias Alecio Mattei, Ohio State

University, USA

Reviewed by:

Jianbo Gao, Wright State University, USA

Abdelmalik Moujahid, University of the Basque Country UPV/EHU,

Spain

\section{*Correspondence:}

Andrei I. Skorinkin, Radioelectronics Department, Institute of Physics,

Kazan Federal University, 16

Kremliovskaja Str., Room 102, Kazan

420008, Russia

e-mail: askorink@yandex.ru
In traditional studies of changes in cell membrane potential or trans-membrane currents a large part of the recorded data presents "a pure noise." This noise results mainly from the random openings of membrane ionic channels. Different types of stationary or non-stationary noise analysis have been used in electrophysiological experiments for identification of channels kinetic states. But these methods have a limited power and often cannot answer to the main question of the experimental study: do external factors induce a significant change of channels kinetics? A new method suggested in the current study is based on the scaling properties of the beta-distribution function that allows reducing the series containing 200,000 and more data points to analysis of only 10-20 stable parameters. The following clusterization using the generalized Pearson correlation function allows taking into account the influence of an external factor and combine/separate different parameters of interest into a statistical cluster considering the influential parameter. This method which we call BRC (Beta distribution-Reduction-Clusterization) opens new possibilities in creation of a largely reduced database while extracting specific fingerprints of the long-term series. The BRC method was validated using patch clamp current recordings containing 250,000 data points obtained from the living cells and from open tip electrode. The numerical distinction between these two series in terms of the reduced parameters was obtained.

Keywords: noise analysis, detrended fluctuation analysis, fluctuation spectroscopy based on beta-distribution, sequence of the ranged amplitudes, membrane currents of neurons

\section{INTRODUCTION}

During electrophysiological studies it is common to record rather long tracks of signals. These signals are registered as temporal variations of cell membrane potential or trans-membrane currents induced by the opening of some ligand- or voltage-gated or even chaotic ionic channels. Usually the principal aim of such a study is the registration of some macroscopic signals—evoked or spontaneous - and the change of parameters of these signals characterizes the total effect of some actions that are located in the experimental object. But a large part of the record forms a so-called "empty track" containing a "pure noise" only. It is well known that this noise reflects mainly the result of random openings of transmembrane ionic channels. Different types of stationary or non-stationary noise analysis have been used for identification of these channels' states (Neher and Sakmann, 1976; Sigworth, 1980, 1985, 1986; Läuger, 1985; Traynelisa and Jaramilloa, 1998; Alvarez et al., 2002; Venkataramanan and Sigworth, 2002).

Unfortunately, these methods have not come into widespread use among physiologists since they often cannot answer the main question of the study: If this drug or this change of environment state induces the reliable change of channels condition or not?

Thus, there is an urgent task to develop a special language that can be compact and reliable in order to describe accurately very long current streams (long-time series) with hidden signals and noise in terms of a finite and statistically understandable set of reduced parameters. In this paper we want to show how to develop this special language based on an example of the analysis of signals recorded in rat's spinal cord slices. Besides this problem we want to show how to detect the presence of the biological object inside the experimental set. For this purpose we also recorded data representing the dependence of the current vs. time when the biological object is absent. Examples of currents recorded in a living cell and with empty electrodes are shown in Figure 1. It is well noticeable that these two signals are apparently very similar. Even though generally distinguishable by an experienced observer the reliability of these differences cannot be numerically evaluated without some special analytic methods.

To the authors' best knowledge one method is basically suitable for quantitative analysis of the different long-time series. This method was introduced by Peng et al. (1994) and nowadays it 

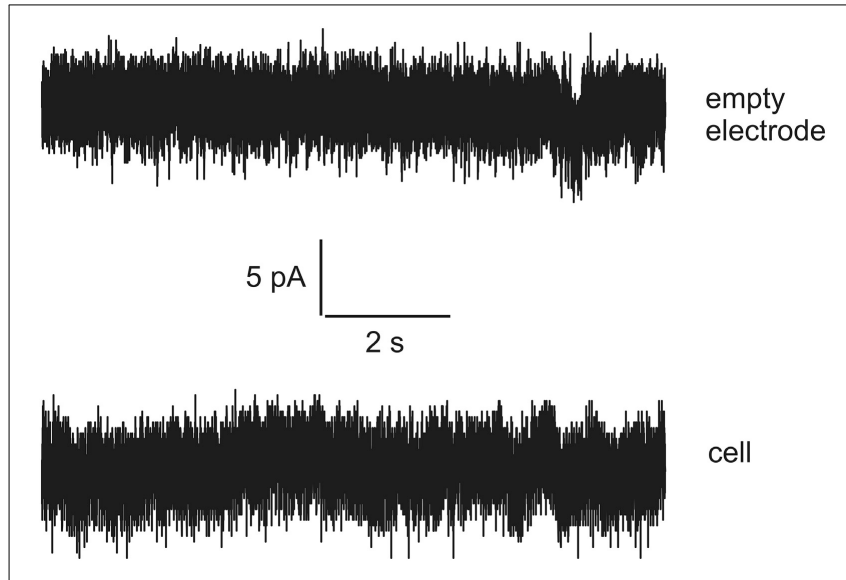

FIGURE 1 | The examples of currents recorded for empty electrode (top) and in a living cell (bottom). Note the high similarity of the tracks.

is known as detrended fluctuation analysis (DFA). It was well described in literature by their creators (Ossadnik et al., 1994; Peng et al., 1995) and found its application in analysis of biomedical (Penzel et al., 2003; Jospin et al., 2007; Burr et al., 2008) and other (Hausdorff et al., 1995, 1996) data. But it is necessary to note that the DFA algorithm works well only for certain types of non-stationary time series (especially having slowly varying trends), it is not designed to handle all possible non-stationarities in real-world data. This algorithm was not free also from uncontrollable errors that are associated with approximate fitting of detrended fluctuations by the segments of straight lines or by the parabolic or high order polynomials (Kantelhardt et al., 2001). The final straight line with power-law exponent $\alpha_{\text {DFA }}$ is obtained as a slope in a double-log scale as a result of the fitting procedure and contains the fitting error that depends also on the type of segmentation of the initial series considered. These uncontrollable errors (usually they are not properly analyzed in the literature) can lead to different results in calculation of the desired value of the $\alpha_{\text {DFA }}$ and other associated fitting parameters in analysis of the same long-time series.

A technique, called scale-dependent Lyapunov exponent (SDLE, see Gao et al., 2006, 2012b, 2013; Hu et al., 2010), provides a more comprehensive characterization of complex time series. Some of DFA's limitations have been overcome recently as well by using a new method called adaptive fractal analysis (AFA, see Gao et al., 2010, 2011, 2012a; Riley et al., 2012; Kuznetsov et al., 2013). AFA has been shown to be able to determine global trends, remove noise, perform fractal analysis and multiscale decomposition and present data as a curve. However, new tools could be developed specifically designed to show and estimate even mild differences between two long time series.

Thus, it would be desirable to have a new method with "high resolution" (10-20 significant parameters) to distinguish more accurately the experimental data and effect of treatments. In this paper we demonstrate such method based on some invariant properties of the beta-distribution function; furthermore this method admits a procedure that controls the error in each stage of its application. From our point of view the effectiveness of new approach is based on the monotone behavior of the primary fitting parameters that admit the secondary fit. This peculiarity allows compressing initial fitting parameters with the help of the secondary fit and present initial data set in more compact form.

The four fitting parameters $(A, B, \alpha, \beta)$ of beta-distribution can be interpreted and used for quantitative reading of fluctuations arising on different scales of the long-time series considered. In previous papers (Nigmatullin, 2010; Nigmatullin et al., 2012) based on the principle of the strong correlation of random sequences it was shown that the cumulative (integral) curve obtained from the sequence of the ranged amplitudes (SRA) can be described with high accuracy by means of the beta-distribution function. In other words, any detrended random sequence being transformed to the SRA (when all amplitudes of the initial sequence are sorted out and located in the descending order $y_{1}>$ $\left.y_{2}>\ldots>y_{N}\right)$ after elimination of its mean value and subsequent integration, forms a bell-like curve $J(x)$ that can be fit (with controllable relative error) by the function:

$$
J(x) \cong J b(x)=A\left(x-x_{0}\right)^{\alpha}\left(x_{N}-x\right)^{\beta}+B
$$

Here the limiting values $x_{0}<x_{N}$ define the ends of the location interval of the random sequence considered. In many cases the parameters $x_{0}, x_{N}$ are known. Other quantitative parameters $(A, B, \alpha, \beta)$ should be found from the fitting procedure of the function $J(x)$ to the curve $J b(x)$. The power-law exponents $(\alpha, \beta)$ reflect the fractal properties of the random sequence considered and the presence of the memory that is expressed in the behavior of the corresponding SRAs. The criterion for the verification of the presence of memory in two random sequences which are compared is as follows. If one SRA being plotted with respect to another one forms a curve close to a straight line then these two random curves are defined as a having a relative memory and can be considered as being strongly correlated. This important property allows transforming any segment of a random sequence to a beta-distribution function and "read" this segment in terms of four unknown fitting parameters $(A, B, \alpha, \beta)$. Such transformation from 30 to 50 or even more initial points belonging to a random sequence can be read in terms of these four parameters only. This allows us to suggest a new type of spectroscopy based on some scaling properties of the beta-distribution. This transformation is called Fluctuation Spectroscopy based on BetaDistribution (FSBD). In general we suggest a method which we call BRC (Beta distribution-Reduction-Clusterization). The basic problem that is solved in this paper by using the BRC method can be formulated as follows: Is it possible to suggest a reliable method with controllable error that has a wide range of applicability and which has a flexible small set (10-20) of statistically understandable parameters for quantitative characterization of the differences between long-time series?

\section{MATERIALS AND METHODS PREPARATION OF SPINAL CORD SLICES}

Ten- to Twenty-days-old Wistar rats were deeply anesthetized with diethyl ether and killed by decapitation. After laminectomy, the spinal cord was excised, and immediately immersed in cold $\left(0 \div 4^{\circ} \mathrm{C}\right)$ artificial cerebrospinal fluid containing (in $\left.\mathrm{mM}\right): 126$ 
$\mathrm{NaCl}, 26 \mathrm{NaHCO}_{3}, 2.5 \mathrm{KCl}, 1.25 \mathrm{NaH}_{2} \mathrm{PO}_{4}, 2 \mathrm{CaCl}_{2}, 2 \mathrm{MgCl}_{2}$, and 10 glucose (bubbled with $95 \% \mathrm{O}_{2}$ and $5 \% \mathrm{CO}_{2} ; \mathrm{pH} 7.3$; 310 mOsm measured). Several transverse slices (250- $\mu$ m thick) were prepared from the lumbosacral enlargement (L4-6) with a vibratome (VT1000S, Leica, Nussloch, Germany).

\section{WHOLE-CELL RECORDINGS}

Slices were transferred to a recording chamber $(300 \div 400 \mu \mathrm{l}$ volume) and continuously superfused with oxygenated artificial cerebrospinal fluid at $3 \mathrm{ml} / \mathrm{min}$ and $22 \div 24^{\circ} \mathrm{C}$. Interneurons were visualized with an upright interference contrast microscope and $\mathrm{a} \times 40$ water immersion objective (Axioscope FS, Carl Zeiss, Oberkochen, Germany). Patch-pipettes (tip resistance, $5 \div$ $7 \mathrm{M} \Omega$ ) were prepared by a puller (Flaming-Brown P97; Sutter, Novato, CA, USA) from borosilicate capillaries and were filled with intracellular solution consisting of (in $\mathrm{mM}$ : potassium gluconate 140, $\mathrm{NaCl} 10, \mathrm{MgCl}_{2}$ 3, HEPES 10, EGTA 11; pH 7.3 adjusted with $\mathrm{KOH} ; 300$ mOsm measured).

Interneurons were voltage-clamped at $-65 \mathrm{mV}$ in the wholecell configuration after obtaining GV seals (usually not less than $2 \mathrm{GV}$ ) by means of a patch-clamp amplifier (Axopatch 200B; Molecular Devices, Sunnyvale, CA, USA). Compensation of capacitance $(\mathrm{Cm})$ and series resistance (Rs) was achieved with the inbuilt circuitry of the amplifier. Series resistance was compensated by $40 \div 70 \%$ and did not change appreciably from the beginning to the end of the experiments, indicating stable recording conditions. The tracks used for comparison were recorded by the immersion of filled patch-pipettes in artificial cerebrospinal fluid; the patch-pipettes were voltage-clamped at $-65 \mathrm{mV}$ too.

Then all data were sampled at $10 \mathrm{kHz}$ and stored on-line with a PC using the pClamp 10.0/Clampex 10.0 software package (Molecular Devices).

\section{SCALING PROPERTIES OF THE BETA-DISTRIBUTION AND DESCRIPTION OF THE TREATMENT PROCEDURE}

In this section we want to demonstrate the scaling properties of Expression (1). We subject $x, x_{0}$ and $x_{N}$ in Expression (1) to the following scaling transformations, keeping the power-law exponents $\alpha$ and $\beta$ invariable: $x=\xi \cdot x^{\prime}+b . x_{0}=\xi \cdot x_{0}^{\prime}+b, x_{N}=$ $\xi \cdot x_{N}^{\prime}+b$, which gives the following beta transformation:

$$
J b(x) \rightarrow J b\left(x^{\prime}\right)=A^{\prime}\left(x^{\prime}-x_{0}^{\prime}\right)^{\alpha} \cdot\left(x_{N}^{\prime}-x^{\prime}\right)^{\beta},
$$

where $A^{\prime}=A \cdot \xi^{(\alpha+\beta)}$. This is the accurate mathematical result that follows from the scaling transformation of the initial coordinates.

In order to have a simple criterion for comparison of the two beta-distributions let us calculate the values of two extreme points $\bar{x}, \bar{x}^{\prime}$ belonging to the functions $J b(x)$ and $J b\left(x^{\prime}\right)$ respectively.

$$
\begin{aligned}
\bar{x} & =w_{1} x_{0}+w_{2} x_{N}, \bar{x}^{\prime}=w_{1} x_{0}^{\prime}+w_{2} x_{N}^{\prime}, \\
w_{1} & =\frac{\beta}{\alpha+\beta}=\frac{x_{N}-\bar{x}}{\Delta}, w_{2}=1-w_{1}, \Delta=x_{N}-x_{0}, \Delta^{\prime}=\frac{1}{\xi} \Delta, \\
\bar{H} & =J b(\bar{x})=A w_{1}^{\beta} w_{2}^{\alpha} \Delta^{\alpha+\beta}+B, \\
\bar{H}^{\prime} & =J b\left(\bar{x}^{\prime}\right)=A^{\prime} w_{1}^{\beta} w_{2}^{\alpha}\left(\Delta^{\prime}\right)^{\alpha+\beta}+B,
\end{aligned}
$$

$$
\bar{H}^{\prime}=J b\left(\bar{x}^{\prime}\right)=A w_{1}^{\beta} w_{2}^{\alpha} \xi^{\alpha+\beta}\left(\frac{1}{\xi}\right)^{\alpha+\beta}+B \equiv \bar{H} .
$$

From Expressions (3) it follows that for the scaling transformation (2) the heights $\bar{H}, \bar{H}^{\prime}$ of the extreme points of the two belllike distributions at the fixed values of the power-law exponents $\alpha$ and $\beta$ and parameter $B$ should coincide with each other.

Besides this criterion it is necessary to take into account the scaling relationship between the heights $\bar{H}, \bar{H}^{\prime}$. If two power-law exponents $\alpha$ and $\beta$ are subjected to the scaling transformation at the fixed value of the length $\Delta=x_{N}-x_{0}$ :

$$
\alpha^{\prime}=\theta \alpha, \beta^{\prime}=\theta \beta
$$

then simple manipulations lead to the second scaling relationship:

$$
\frac{\bar{H}^{\prime}}{A^{\prime}}=\left(\frac{\bar{H}}{A}\right)^{\theta}
$$

Here the amplitudes $A$ and $A^{\prime}$ are defined by relationships (1) and (2), respectively. The consideration of the scaling properties of the beta-distribution allows one to suggest the following two steps.

Step 1. This step includes the formation of the sequence of the range amplitudes (SRA) when all amplitudes located on the fixed length $\Delta=x_{N}-x_{0}$ are ordered in descending order $y_{1}\left(x_{0}\right)>$ $y_{2}>\ldots>y\left(x_{N}\right)$.

Step 2. Numerical integration of the SRA with respect to its mean value and subsequent fit to the function (1).

Figure 2 illustrates this transformation which is realized after application of these two steps.

Each sub-segment having equal length $\Delta$ is transformed to its SRA (Figure 2A) in Step 1, and the integration of the SRAs with respect to its subtracted mean value gives finally the desired bell-like curve that can be fit to Expression (1) in Step 2. Mathematically these two steps correspondingly are expressed as:

$$
\begin{aligned}
\operatorname{SRA}\left(y\left(x_{j}\right)\right) & =\operatorname{sort}\left(y\left(x_{j}\right)\right) \rightarrow \Delta \operatorname{SRA}\left(y\left(x_{j}\right)\right) \\
& =\operatorname{SRA}\left(y\left(x_{j}\right)\right)-\frac{1}{\Delta} \sum_{j=1}^{\Delta} \operatorname{SRA}\left(y\left(x_{j}\right)\right) \\
& \equiv \operatorname{SRA}\left(y\left(x_{j}\right)\right)-\langle\ldots\rangle
\end{aligned}
$$

Here the integer index $j(j=1,2, \ldots, N)$ numerates the number of data points in the fixed segment $\Delta=x_{N}-x_{0}$ containing initially 30-50 data points.

$$
\begin{aligned}
J\left(x_{j}\right)= & J\left(x_{j}\right)+\frac{1}{2}\left(x_{j}-x_{j-1}\right) \cdot\left(\Delta \operatorname{SRA}\left(y\left(x_{j}\right)\right)\right. \\
& \left.+\Delta \operatorname{SRA}\left(y\left(x_{j}\right)\right)\right), J_{0}=0 .
\end{aligned}
$$

Figure 2 demonstrate the realization of these two steps [with the usage of Expression (6)] on a short segment belonging to the membrane current initial time segment (containing 250,000 data points). We should notice that the mean value $\langle\ldots\rangle$ of the chosen segment should be subtracted and the integration procedure [the last row in (6)] should be realized with the help of the 


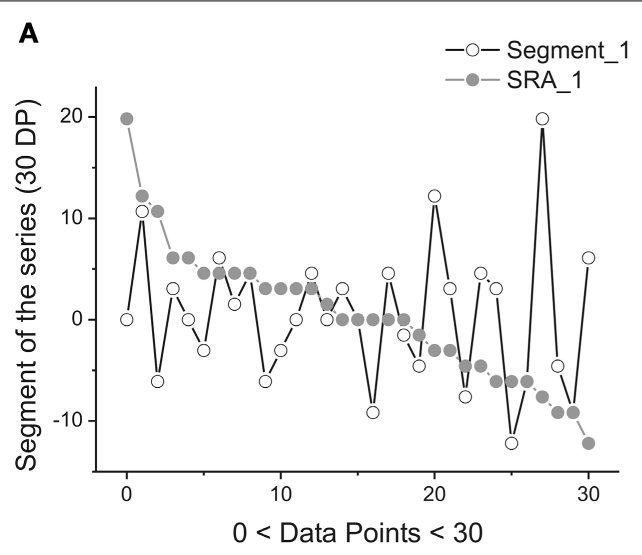

FIGURE 2 | Example of one segment (marked as Segment_1) containing 30 points. (A) The sequence of the ranged amplitudes (SRA) given in descending order and marked by gray stars. On vertical axes the values of the current are given in picoampers. (B) The bell-like curve (marked by crossed stars) obtained

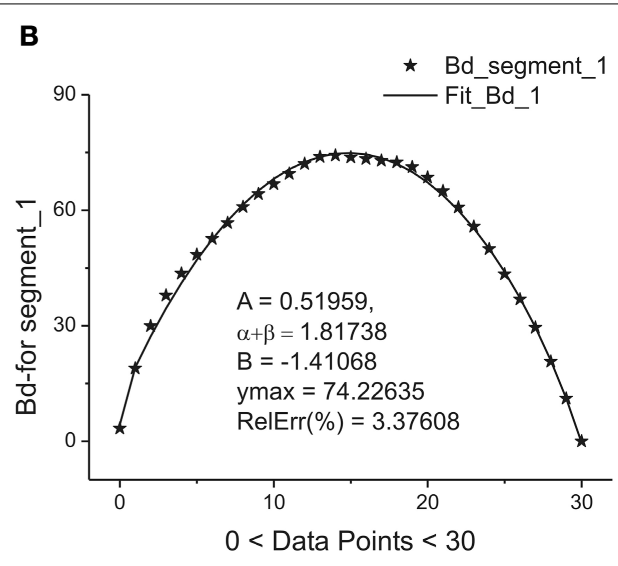

by integration from SRA_1 (shown on the previous $\mathbf{A}$ by gray stars) and its fit marked by the bold solid line. The fitting parameters of this curve are given inside of this figure. As it follows from this figure 30 data points are sufficient for providing the acceptable fit with the value of the relative error close to $3.5 \%$. trapezoid method. As a result of calculation of Expression (6) we obtain the desired bell-like curve $J\left(x_{j}\right)$.

Figure 2B shows the quality of the fitting of the bell-like curve obtained to the beta-distribution. In order to have the value of the relative error:

$$
\begin{aligned}
\text { RelErr } & =\left(\frac{\operatorname{stdev}(J(x)-J b(x))}{\operatorname{mean}(J(x))}\right) \cdot 100 \% \\
\text { where stdev }(f(x)) & =\left[\frac{1}{N_{\Delta}} \sum_{j=1}^{N_{\Delta}}\left(f\left(x_{j}\right)-\text { mean }(f(x))\right)^{2}\right]^{1 / 2} \\
\text { mean }(f(x)) & =\frac{1}{N_{\Delta}} \sum_{j=1}^{N_{\Delta}} f\left(x_{j}\right)
\end{aligned}
$$

to be limited to a few percentages $(2-5) \%$ we should choose the length of the minimal segment $\Delta_{\min }$ of the initial series containing initially 30-50 data points. In Expression (7) the value $N_{\Delta}$ defines the number of data points that enters in the segment of the length $\Delta$. Thus, the first reduction criterion should be written as:

$$
\Delta_{\text {min }} \cdot \xi^{k}=N_{\text {total }}
$$

Here the scaling parameter $\xi$ has the same meaning as in Expression (2).

This requirement allows one to consider the long-time series containing the total number of data points $\left(j=1,2, \ldots, N_{\text {total }}\right)$ in terms of the reduced parameters of the beta-distribution $(A, B, \alpha$, $\beta$ ) depending on parameter $k$. Further it is convenient to rewrite condition (8) in the following form changing the numeration of the current parameter $k$ :

$$
\Delta_{k}=\frac{N_{\text {total }}}{\xi^{K+1-k}}, \quad k=1,2, \ldots, K+1,
$$

where [in comparison with (8)] the value $\Delta_{1}$ should coincide with the minimal value $30<\Delta_{\min }<50$ giving the condition for finding the limiting value of $\mathrm{K}$ (the total number of segments is equaled to $K+1$ ). In the opposite case, the value $\Delta_{K+1}$ should give the maximal length coinciding with the value $N_{\text {total }}$. As a result of this reduction procedure one can transform $N_{\text {total }}$ data points to $4 .(K+1)$ parameters. But this step is not sufficient. If the functions $A_{k}, B_{\mathrm{k}},(\alpha+\beta)_{\mathrm{k}}$ have monotonic behavior one can realize further reduction to the primary set of the fitting parameters describing these functions.

Now it is necessary to explain why the sum of the parameters $(\alpha+\beta)$ is selected instead of considering each-power law exponent separately. This selection is based on the comparison of these exponents with the single power-law exponent $\alpha_{D F A}$ figuring as the basic parameter in the DFA. It is easy to see that relationship $\alpha+\beta=1$ with $\alpha \approx \beta \approx 0.5$ (for this case betadistribution looks like a semicircle) corresponds to a distribution with the absence of power-law correlations in the time series. From another side it gives for $\alpha_{D F A}=0.5$. Comparison with these two power-law exponents leads us to the following approximate expression:

$$
\alpha_{D F A} \cong \frac{1}{2}(\alpha+\beta)
$$

One can notice also that Expression (10) does not contradict other well-known power-law exponents (Hausdorff et al., 1995; Burr et al., 2008) $\beta_{f}=2 \alpha_{D F A}-1$ that is used for description of the power-law spectrum $S(f) \sim f_{f}^{-p}$ and decay of autocorrelation function $C(t)=\left\langle x_{i} x_{i+1}\right\rangle \sim t^{-1}$ with $\gamma=2-2 \alpha_{D F A}$. From the requirements $\left(\beta_{f}, \gamma>0\right)$ it follows that:

$$
1 \leq(\alpha+\beta)=2 \alpha_{D F A} \leq 2
$$

We want to stress here that this requirement is approximate and can serve as an indication for division of long-time series with fractal structure (because it does not contradict with wellknown inequalities) known before from series with self-similar structure. 
The left-hand inequality follows from the requirement $\beta_{f}>0$ and does not contradict with numerical results obtained in other papers (Penzel et al., 2003; Jospin et al., 2007; Burr et al., 2008). We should also note that the equality $(\alpha+\beta)=2$ corresponds to a uniform amplitude distribution. The uniform distribution leads to the degeneration of the corresponding SRA to a straight line (Nigmatullin, 2010). The beta-distribution in this case is described by a parabolic curve. If one of the power-law exponent (say $\alpha \rightarrow 0$ ) then the position of extreme point $\bar{x} \rightarrow x_{0}$. Because of normalization $w_{1}+w_{2}=1 \beta \rightarrow 1$. This statement is valid also in the opposite case when $\alpha \rightarrow 1, \beta \rightarrow 0$. So, the last relationship (11) can be considered as a specific fractal test in our further calculations. Here we should also note that in practical applications the existence of the interval $0<\alpha+\beta<1$ and inequality $\alpha+\beta>2$ also are possible. For the first case, for small values of $\alpha$ and $\beta$ the beta-distribution degenerates to a rectanglelike curve. In the second case the values of the derivatives on the ends $\left(x_{0}, x_{N}\right)$ of the beta-distribution have zero values. These two cases correspond to degeneration of the fractal properties of the time-series analyzed. The verification of relationship (11) on the Weierstrass-Mandelbrot function that represents itself the selfaffine function (see its definition in Feder, 1988) confirms the relationship (11). So, for practical purposes it is useful to work with the combination of $(\alpha+\beta)$.

The statistical and geometrical meaning of other parameters entering to (1) can be explained as follows. The value of the amplitude $A$ together with the height $H$ of the beta-distribution is associated with intensity of the fluctuations analyzed. As one can see from Figure 3A the angle of the SRA slope counted off from zero point (after elimination of its mean value) is proportional to the height of the corresponding fluctuation that is expressed in the form of a beta-distribution in Figure 3B. If this angle approaches the vertical axis, the height of the distribution becomes large. In the opposite case when this angle tends to zero the height of the distribution is small. See Figure 3B where the first 14 beta-distributions are shown. The measure of asymmetry can be connected with parameters $B$ and the values of weight factors $w_{1,2}$ that are defined by Expression (3). The value $w_{1}=$ 0.5 corresponds to the complete symmetry of the distribution in the horizontal direction. Any shift of this parameter to the left$\left(w_{1}<0.5\right)$ or to the right-hand side $\left(w_{1}>0.5\right)$ reflects the horizontal asymmetry of the distribution. A small asymmetry of this distribution in vertical direction is controlled by the parameter $B$.

Step 3. After selection of the scaling parameter $\xi$ and the limiting value $K$ from Expression (9) one can obtain a family of bell-like curves that can be fitted to Expression (1). The calculated fitting parameters $A_{k}, \alpha_{k}, \beta_{k}, B_{k}, k=1,2, \ldots, K+1$ from Expression (1) are obtained. The set of these bell-like curves and the corresponding fitting parameters forms the total fluctuation spectrum based on the beta-distribution (FSBD). Each part of this FSBD contains the corresponding beta-distribution:

$$
J b_{k}\left(x_{j}\right)=A_{k}\left(x_{j}-x_{0, k}\right)^{\alpha_{k}}\left(x_{N, k}-x_{j}\right)^{\beta_{k}}+B_{k} .
$$

Step 4. In order to subject them to the scale-invariant properties described above it is necessary to average this family of distributions and consider only one weighted distribution:

$$
\begin{aligned}
\left\langle J b_{k}\left(x_{j}\right)\right\rangle & =\frac{1}{N B d_{k}} \sum_{j=1}^{N B d_{k}} J b_{k}\left(x_{j}\right), \quad j=1,2, \ldots, N B d_{k}, \\
N B d_{k} & =\frac{N_{\text {total }}}{\Delta_{k}}
\end{aligned}
$$

located in the given interval $\Delta_{k}$. Here the parameter $N B d_{k}$ coincides with number of beta-distributions calculated for the given $k$. Figure 4 shows the averaged beta-distribution obtained for the cell number 3. If $N_{\text {total }}=250,000$ then from condition (9) at the given $\Delta_{1}=32$ and $\xi=2$ we obtain that $K=13$. So, the total number of beta-distributions $N B d_{1}=N_{\text {total }} / \Delta_{1}=8333$. The first 14 distributions belonging to this family is shown in Figure 3B.

Step 5. Further calculations are reduced to the analysis of the functional dependencies $A_{k}, \alpha_{k}, \beta_{k}, B_{k}, k=1,2, \ldots, K+1$ with

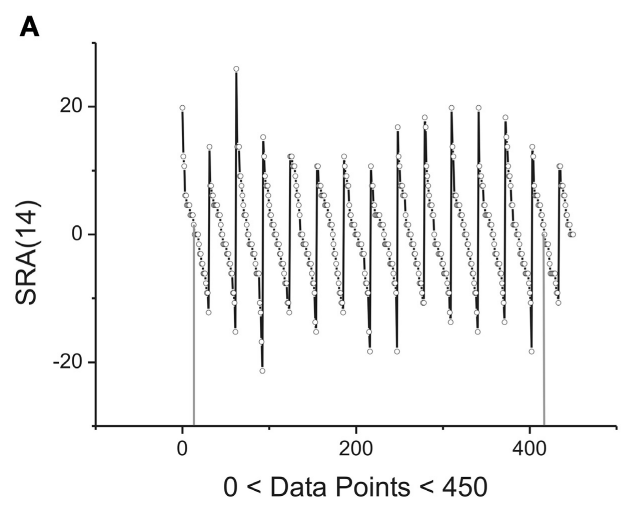

FIGURE 3 | Example of first 14 segments, each segment contains 30 points. (A) The first 14 SRAs calculated for the large-time membrane current sequence containing in total 250,000 data points. After elimination of their mean values (two limiting of them are shown by vertical gray lines) and subsequent integration one can obtain a family of the bell-like

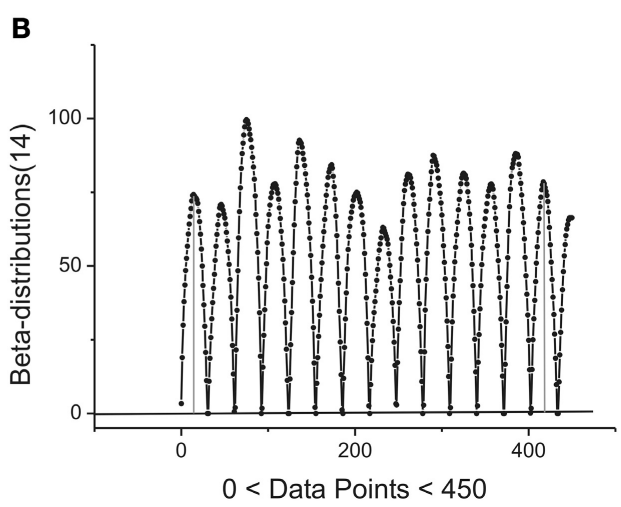

curves. They are shown below. (B) The first 14 beta-distributions obtained by numerical integration from the SRAs given of the previous panel. For the total sequence having 250,000 data points we have in general 8333 distributions of such kind. Two limiting heights are marked by solid gray lines. 


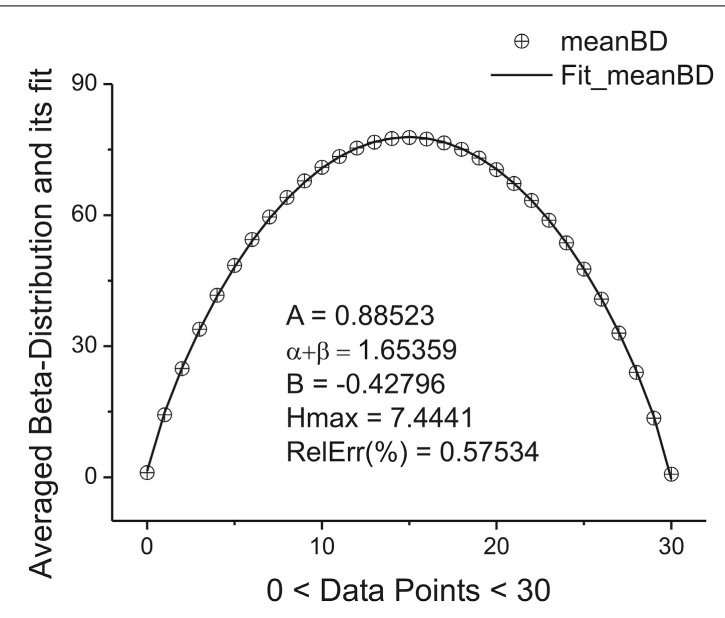

FIGURE 4 | The averaged beta-distribution (averaged with the usage of Expression (13) for the given $N B d_{1}=N_{\text {total }} / \Delta_{1}=8333$ ). The fitting parameters are shown inside of this figure.

respect to the variable $k$. We define them as the primary fitting parameters characterizing the averaged distribution (13). Further analysis shows that the amplitude $A_{k}$ has monotonic behavior and can be described by a simple exponential behavior:

$$
\left\langle A_{k}\right\rangle=A_{1} \cdot \exp \left(\lambda_{a} \cdot k\right)+A_{0} .
$$

Preliminary calculations show that this monotonic behavior is conserved for the long-time series without any trend. The presence of trend distorts this behavior.

This dependence follows after substitution of Expression (9) in relationship (2) for the amplitudes. The perfect fit of this monotone curve is shown in Figure 5A. Other dependencies are not so simple but nevertheless they can be identified from simple power-law and exponential hypothesis with the help of the eigencoordinates (ECs) method (Baleanu et al., 2011; Ciurea et al., 2011). The dependences $<(\alpha+\beta)_{\mathrm{k}}>\equiv S_{\mathrm{k}}(\alpha \beta)$ and $<B_{k}>$ have also monotonic character and can be fitted by means of two simple functions:

$$
\begin{aligned}
S_{k}(\alpha \beta) \cdot k^{\nu} & =A_{p l} \cdot k+B_{p l}, \\
\left\langle B_{k}\right\rangle & =B_{1} \cdot \exp \left(\lambda_{B} \cdot k\right)+B_{0}
\end{aligned}
$$

These functions are shown, respectively, in Figures 5B,C. So, finally we obtain 10 fitting parameters that can be combined with 9 parameters figuring in Expressions (14) and (15) $\left[\lambda_{\mathrm{a}}, A_{1}, A_{0}\right]$, $\left[\nu, A_{p l}, B_{p l}\right],\left[\lambda_{B}, B_{1}, B_{0}\right]$ and the limiting value of parameter $w_{1}, \mathrm{~K}+1$. The behavior of this weight factor is shown in Figure 5D.

These ten parameters can be used as the primary set of the fitting parameters for creation of a specific "fingerprint" of the long-time series considered. The idea of clusterization of these parameters is discussed in Results Section. Further analysis shows that the distribution of the heights and mean values of the SRAs obtained for the family of distributions at $\Delta_{1}$ also forms two other different beta-distributions. These distributions are important also for clusterization purposes because initially the information about the secondary distribution of the heights of the initially formed beta-distributions family and mean values of the corresponding SRA were not taken into account. The distributions of the heights and mean values together with their beta-distributions are shown in Figures 6, 7, correspondingly. After fitting of these two distributions one can obtain in addition 5 significant parameters characterizing each beta-distribution separately.

$$
\begin{array}{r}
{\left[A_{H},(\alpha+\beta)_{H}, w_{1, H}, \max \left(B d_{H}\right), \operatorname{mean}\left(S R A_{H}\right)\right]} \\
{\left[A_{m n},(\alpha+\beta)_{m n}, w_{1, m n}, \max \left(B d_{m n}\right), \operatorname{mean}\left(S R A_{m n}\right)\right] .}
\end{array}
$$

These ten additional parameters we define as the secondary fitting parameters. The statistical meaning of these parameters are the following. The parameters $A_{H, m n}$ characterize the amplitudes of beta-distributions referring, correspondingly, to the heights $(H)$ and mean values $(m n)$. The sum $(\alpha+\beta)_{H, m n}$ contains the information about their power-law exponents, $w_{1, H, m n}$ gives the information about their asymmetry, $\max \left(B d_{H}, B d_{m n}\right)$ signifies their heights, and the fifth parameter $\mathrm{SRA}_{H, m n}$ contains information about the mean values of these two additional distributions.

From our point of view, these 20 (10 primary and $10 \mathrm{sec}-$ ondary) significant parameters [figuring in Expressions (14)(16)] combined together can completely characterize the behavior of fluctuations associated with the long-time series analyzed and containing $N_{\text {total }}=2.5 .10^{5} \div 10^{6}$ and even more data points.

\section{CLUSTERIZATION OF FINAL PARAMETERS BASED ON THE GENERALIZED PEARSON CORRELATION FUNCTION}

For clusterization purposes one can suggest more accurate selection of similar sequences based on internal correlations. For this aim we introduce the generalized Pearson correlation function (GPCF) (Nigmatullin, 2010; Nigmatullin et al., 2012).

$$
G P C F_{p}=\frac{G M V_{p}\left(s_{1}, s_{2}\right)}{\sqrt{G M V_{p}\left(s_{1}, s_{1}\right) \cdot G M V_{p}\left(s_{2}, s_{2}\right)}},
$$

where expression:

$$
\begin{aligned}
& G M V_{p}\left(s_{1}, s_{2}, \ldots, s_{K}\right)= \\
& \left(\frac{1}{N} \sum_{j=1}^{N}\left|n r m_{j}\left(s_{1}\right) \cdot n r m_{j}\left(s_{2}\right) \cdot \ldots \cdot n r m_{j}\left(s_{K}\right)\right|^{m o m_{p}}\right)^{1 / m o m_{p}}
\end{aligned}
$$

determines the generalized mean value $(G M V)$-function of the $K$-th order. Here the generalized mean value (GMV) function determines the mean value for all range of the moments (see Expression (19) below). The set of parameters $\left(s_{1}, s_{2}, \ldots, s_{K}\right)$ determines the type of the random sequence compared. The GPCF determined by Expression (17) coincides with the conventional definition of the Pearson correlation coefficient at $\operatorname{mom}_{\mathrm{p}}=1$. The set of moments are determined by the following expression:

$$
\begin{array}{r}
m o m_{p}=\exp \left(L n_{p}\right), L n_{p}=m n+\left(\frac{p}{P}\right) \cdot(m x-m n), \\
p=0,1, \ldots, P .
\end{array}
$$




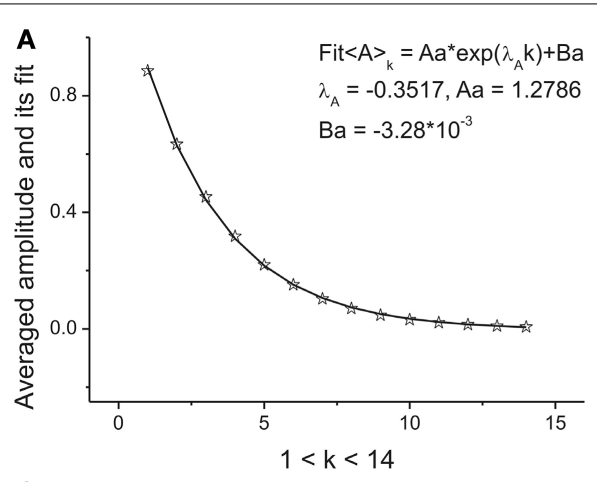

C

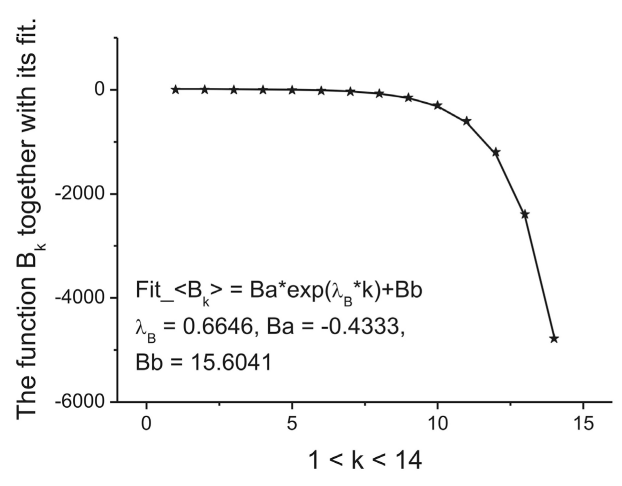

FIGURE 5 | The fitting curves of four parameters. (A) The fit of the amplitude obtained for the averaged beta-distributions for different values of k. See Expression (14) for details. The fitting parameters of the exponential function are given above of this figure. (B) The fit of the function $S_{k}(\alpha \beta)=$ $(\alpha+\beta)_{k}$ defined by Expression (15). Being separated by the power-law exponent with $v=0.955$ it represents the perfect straight line. The slope and intercept of this line are given above of this figure. (C) The fit of the
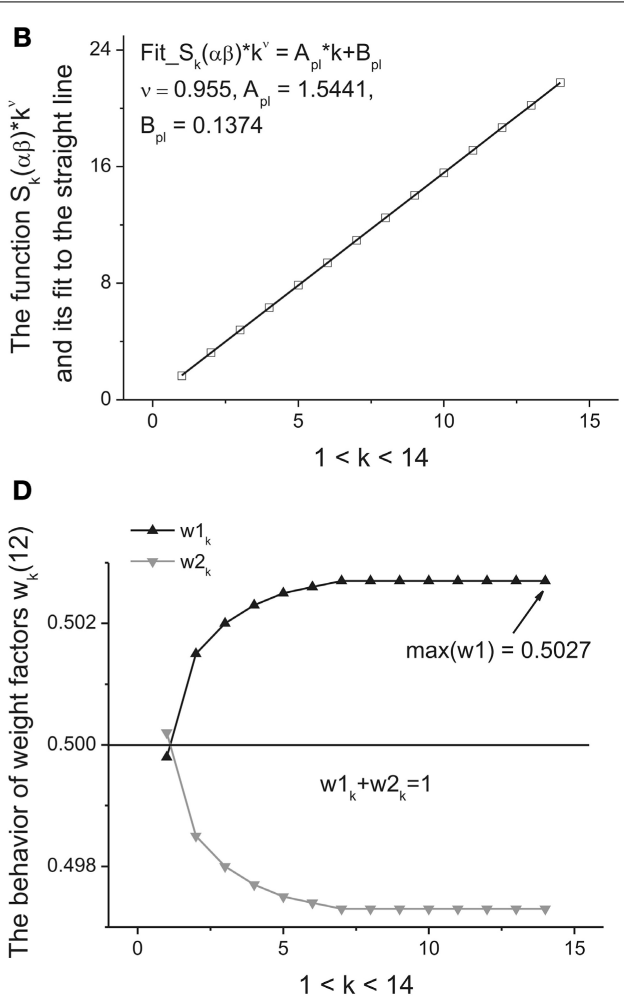

monotonic decreasing function $\left\langle B_{k}>\right.$ defined by Expression (15). The three fitting parameters of this function can be added to the previous ones for characterization of the given long-time series. (D) The behavior of the weight factors with respect to the parameter $k$. As the significant factor characterizing the behavior of the long-time sequence we use the maximal value $\max \left(w_{1}\right)=0.5027$. So, from analysis of the Figure $\mathbf{4}$ and in this figure we can extract 10 primary fitting parameters.
The value mom $_{p}$ in (19) corresponds to the current moment from the interval $[0, P]$. The value $P$ determines the final value of the linear function $L n_{p}$ located in the interval $[m n, m x]$. The values $m n$ and $m x$ define correspondingly the limits of the moments in the uniform logarithmic scale. In many practical cases these values are chosen as $m n=-15, m x=15$ and $P$ is chosen as an integer value located in the interval $[50 \div 100]$. This empirical choice is related to the fact that the transition region of the random sequences considered and expressed in the form of the $G M V$-functions is concentrated usually in the interval $\operatorname{Ln}_{p} \in$ $[-5,5]$. The extended interval $[-15,15]$ is taken usually for calculation of the limiting values of this function in the space of the fractional moments. The initial sequences are chosen in that way: the minimum of the GMV-function coincides with zero value while the upper value of this function coincides with the maximal value of the random sequence considered. In formula (18) the random sequence is normalized to the unit value in accordance with Expressions $(A)$ and $(B)$ :

$$
\begin{aligned}
(A) n r m_{j}(y) & =\frac{y_{j}^{(+)}}{\max \left(y_{j}^{(+)}\right)}-\frac{y_{j}^{(-)}}{\min \left(y_{j}^{(-)}\right)}, \\
y_{j}^{( \pm)} & =\frac{1}{2}\left(y_{j} \pm\left|y_{j}\right|\right)
\end{aligned}
$$

$$
\begin{aligned}
\text { (B) } \operatorname{nrm}_{j}(y) & =\frac{\Delta y_{j}}{\max \left(\Delta y_{j}\right)}, \Delta y_{j}=y_{j}-\min \left(y_{j}\right) . \\
j & =1,2, \ldots, N, \quad 0<n r m(y)<1 .
\end{aligned}
$$

Here, as it was done above, the set $y_{j}$ defines an initial random sequence that can contain a trend or can be compared with another trendless sequence. The symbol $|\ldots|$ and index $j(j=1.2, \ldots, N)$ determine the absolute value and number of the measured points, correspondingly. The second case $(B)$ in $[20(b)]$ corresponds to the case when the initial sequence is positive. If the limits $m n$ and $m x$ in (20) have opposite signs and accept sufficiently large values, then the GPCF function has two plateaus (equaled unit at small numbers of $m n$ (i.e., $G P C F_{m n}=$ 1) and another limiting value $G P C F_{m x}$ depends on the degree of internal correlation between two random sequences compared. This right-hand limit (defined as $L m$ ) is located between two values:

$$
M \equiv \min \left(G P C F_{p}\right) \leq L m \equiv G P C F_{m x} \leq 1 .
$$

The appearance of two plateaus implies that all information about possible correlations is complete and further increasing of the limiting numbers $(m x, m n)$ figuring in (19) is useless. Numerous 


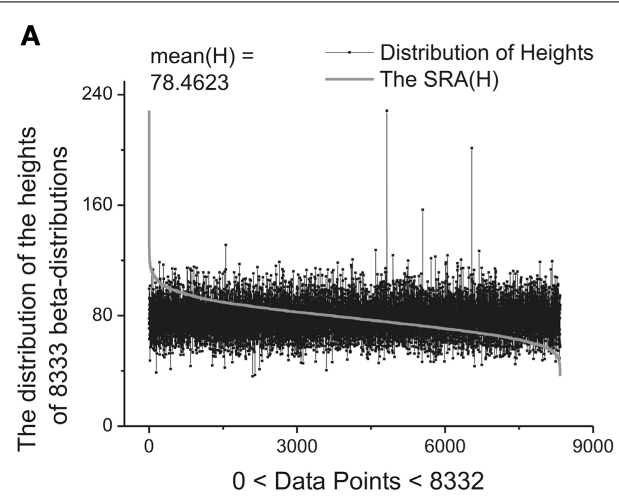

FIGURE 6 | The distribution of the heights of 8333 beta-distributions (when each distribution occupies only 30 data points). (A) Subtracting the mean value of this distribution [mean $(H)=78.4623$ ] one can obtain the bell-like curve again. This curve can be fitted it to the secondary beta-distribution corresponding to the distribution of

\section{$\mathbf{B}$}

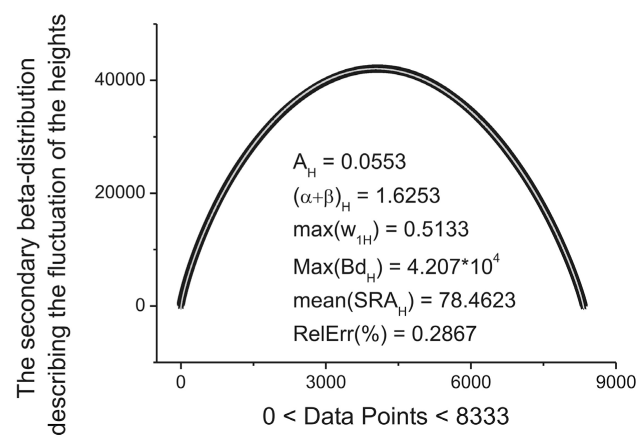

fluctuations of the heights. (B) The fit to beta-distribution function corresponding to fluctuations of the heights. The five fitting parameters of this distribution (shown inside of this figure) can be used as the statistically significant parameters for characterizing of the long-time series considered.

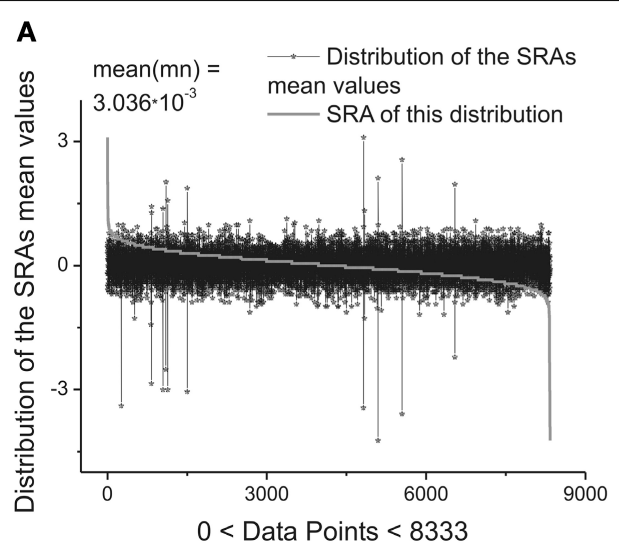

FIGURE 7 | The distribution of the mean values of 8333 beta-distributions (when each distribution occupies only $\mathbf{3 0}$ data points.) that were calculated in the initial analysis. (A) Subtracting the mean value of this distribution (mean $(\mathrm{mn})=3.036 .10^{-3}$ ) one can obtain again the bell-like curve. This curve can be fitted it to the secondary beta-distribution corresponding to the distribution of mean values. (B) The fit to beta-distribution function corresponding to the fluctuations of the

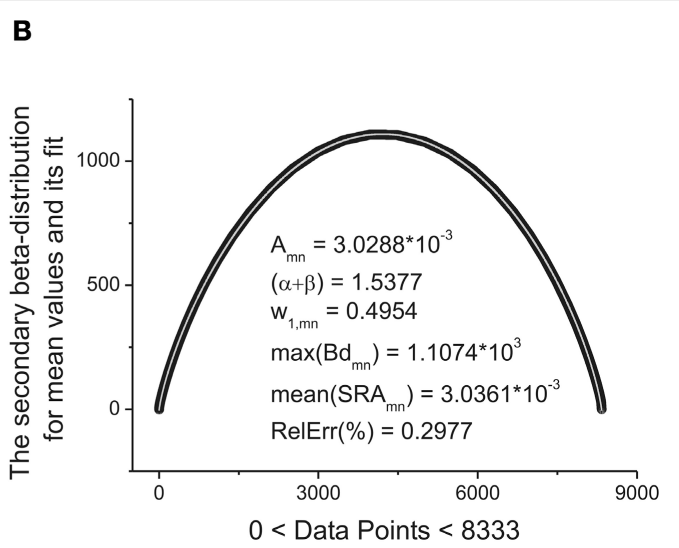

mean values. This information was lost at the preliminary analysis. The five fitting parameters of this distribution (shown inside of this figure) can be used as the statistically significant parameters for characterizing of the long-time series considered. So, in the results of this complete analysis one can obtain 20 statistically significant parameters that can be used for the detailed classification of the long-time series containing $2.5 .10^{5} \div 10^{6}$ data points. tests showed that the high degree of correlations between two random sequences is achieved when $L m=1$, while the lowest correlations are observed when $L m=M$. This empirical observation, having a general character for all random sequences, allows us to introduce new correlation parameter CC (complete correlation)_factor, which is determined as:

$$
C C=M \cdot\left(\frac{L m-M}{1-M}\right) .
$$

We would like to stress here that this factor is determined on the total set of the fractional moments located between $\exp (m n)$ and $\exp (m x)$. As it was mentioned above, in practical calculations for many cases it is sufficient to put $m n=-15$ and $m x=+15$. The CC-factor accepts the unit values when the degree of correlation is high while the case $L m=M$ corresponds to the lowest (remnant) degree of correlations that can be observed between the compared random sequences. In addition, we want to stress also the following fact. This CC-factor does not depend on the amplitudes of the random sequences. The pair random sequences compared should be normalized to the interval: $0 \leq\left|y_{j}\right| \leq 1$. It reflects the internal structure of correlations of the compared random sequences based presumably on the similarity of their probability distribution functions that are not known in many cases. Recent example related to application of the statistics of the fractional moments was considered in paper (Nigmatullin et al., 2012). So, the CC-factor (22) can be used for clusterization of the significant parameters based on the following idea. For a set of significant parameters referring to one qualitative factor one can calculate the limits of CC-factor: 


$$
c f_{\min } \leq C C \leq 1 .
$$

Here the low correlation limit $c f_{\min }$ is determined by the sampling volume and conditions of experiment that should be almost the same for two qualitative factors compared (control/influence of another qualitative factor).

\section{RESULTS}

\section{PROCESSING OF THE LONG-TIME MEMBRANE CURRENT SERIES}

In previous Section we described in details (S1-S5) basic steps of treatment of an arbitrary long-time series. Here we want to make some general remarks related to this procedure. If the long-time series considered contains the clearly expressed but random trend then its random behavior can disturb the monotonic behavior of the primary 9 parameters figuring in the fitting functions (15) and (16). In this cases one can recommend to apply the POLS (procedure of the optimal linear smoothing) described in papers (Baleanu et al., 2011; Ciurea et al., 2011; Nigmatullin et al., 2012) or simple numeric differentiation. These two procedures help to suppress the hidden random trend and obtain the monotonic behavior for the 9 parameters figuring in (15) and (16). In the shown figures we used the scaling factor $\xi=2$. For the rational values of $\xi$ from the interval $(1,2)$ Expression (9) can be modified as:

$$
\Delta_{k}=\frac{N_{t o t}}{\exp [(K+1-k) \ln (2) \cdot \mu]}, \mu=\frac{\ln (\xi)}{\ln (2)} .
$$

So, numerical calculations realized at $\xi=1.5$ show that results are not changed essentially, only the integer variable $k$ in Expressions (15) and (16) is replaced as $k \rightarrow \mu k$. We think that this method has a wide range of its applicability and these two modifications can be taken into account in order to express the long-range time series in terms of 20 significant parameters. In similar manner as it was treated the membrane currents for the randomly taken interneuron-3 one can treat other long-time series related to other $(1,2,4,5,6,7)$ interneurons. Besides, in order to differentiate these random sequences recorded without presence of a biological object we treated in the same manner 6 random sequences corresponding to empty electrode.

The next problem is associated with the finding of criterion of clusterization that helps to combine these "control" membrane currents to one strongly-correlated cluster based on the values of the significant parameters. For each cell these parameters are collected in Table 1. For 6 files corresponding to pure solute (without presence of the cell) the results are collected in Table 2. How to differentiate these 20 quantitative parameters (in this case a qualitative factor is associated with the presence/absence of a biological cell) from each other? The simplest classification can be related to calculation of the mean value and standard deviation of the calculated significant parameter in each row. But more effective scheme of clusterization based on the statistics of the fractional moments and the usage of the complete correlation factor is considered in the next section.

For the clusterization of final parameters we have used new correlation parameter CC described in "Materials and Methods" section Expression (22). The calculation of the CC-factor (in our case it is based on a set of membrane currents associated with 3 "control" measurements for each chosen cell from the total set of currents representing other 7 biologic cells) which is considered as the complex correlation matrix (see Table 3 ) having minimal dimension $(7 \times 7)$ leads to the minimal value $c f_{\min }=0.9238$. The result is not changed essentially if one calculates numerically the corresponding integrals with respect to their normalized significant parameters and then considers their CCfactors. The tendency of the strong correlations between columns of Table 1 is conserved, only the boundary of the correlation interval is slightly increased achieving the value $J c f_{\min }=0.9736$. So, using the method of clusterization based on the statistics of the fractional moments and Expression (22) one can say that all "control" currents measured for the sampling $7 \times 7=49$ form the strongly-correlated cluster with limits $[0.9238,1]$ for the initial set of significant parameters (20 parameters for each sampling) and $[0.9736,1]$ (for the corresponding integrals that are obtained by direct trapezoid method from the normalized significant parameters). In accordance with this method of clusterization one can make the following conclusion: if any another series having 20 significant parameters will give the CC-factor located in the interval $[0.9238,1]$ then it can be considered as the "friend" file belonging to this cluster, in the opposite case it can be considered as a "strange" file. For more reliable identification the saying above can be referred to the integrated columns formed from 20 normalized significant parameters. In the same manner we treated the files corresponding to the electrode currents recorded in normal saline solution without presence of biological object. The 20 desired parameters for 6 files are collected in Table 2. Their correlation matrix presented by Table 4 form another cluster. But attempt to combine the currents corresponding to the living $\mathrm{o}$ cells with currents corresponding to empty electrodes located in saline solution is unsuccessful. If we compare the correlation matrix of Table 5 with the previous ones (Tables 3,4 ) then one can notice that the last matrix is uncorrelated (all elements are close to zero). It means that the presence of the biologic cell completely changes the statistical structure of the current and from qualitative point of view the long-time random sequences of currents recorded for both cases (presence/absence of biological cell) are different.

So, new clusterization method helps to express quantitatively the internal factor as the presence/absence of the living cell (compare this statement with series shown on Figure 1 where the corresponding currents look similar to each other). Definitely, more accurate measurements are needed in order to differentiate from many mixed factors that form a time-series for biological and non-biological objects a specific predominant factor that plays an essential role in this differentiation. But this problem merits a separate research.

\section{DISCUSSION}

It is well known that cellular membrane is the element which largely provides cell functioning. Cell membrane has so many functions that it is difficult even to list-anyone can find them all in each textbook on cell biology. In general the membrane provides all interaction of the cell with the external environment including the perception of the effect of active substances. Withal 
Table 1 | The collection of 20 significant parameters calculated for 7 cells based on calculation of registered membrane currents.

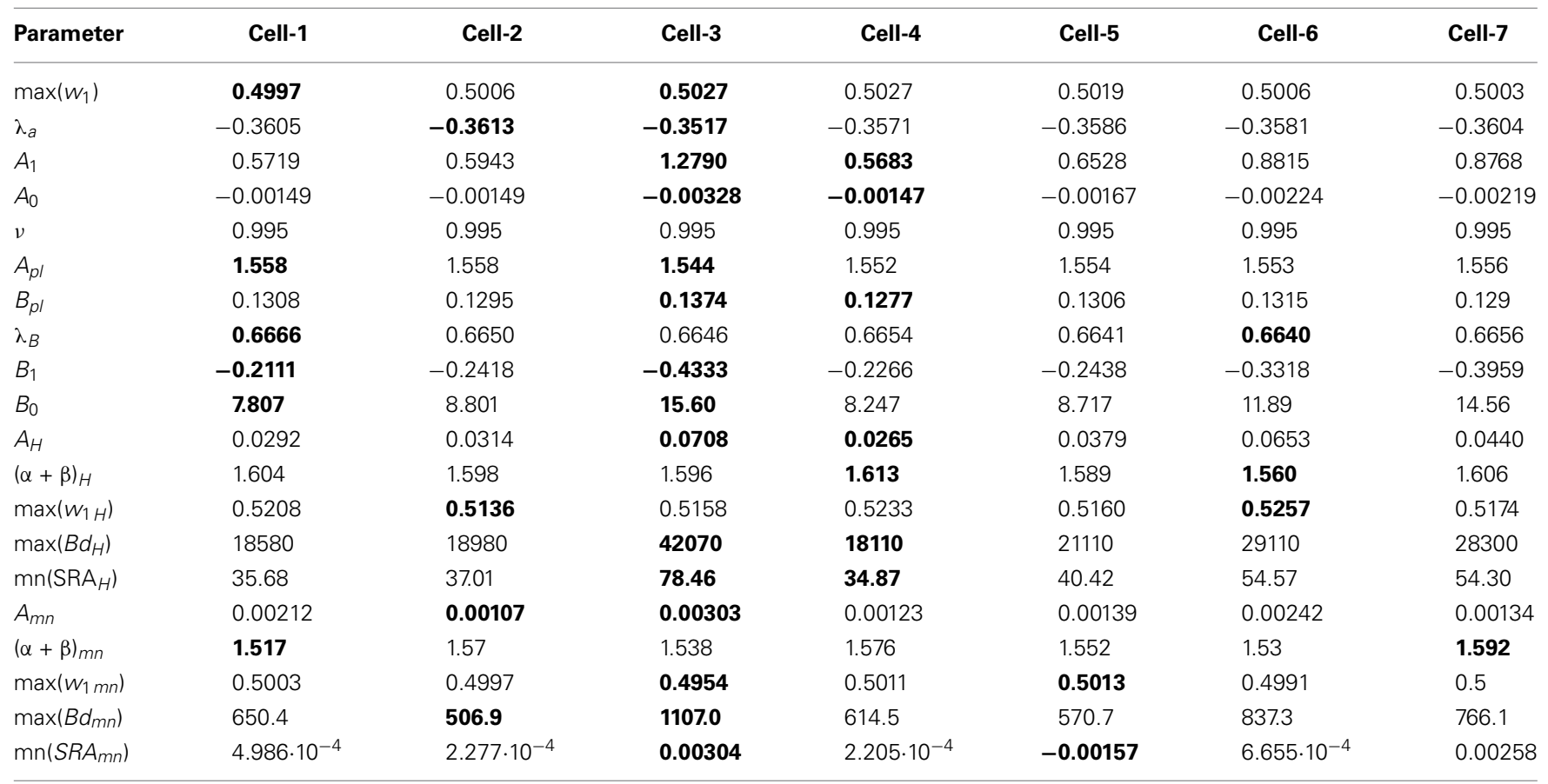

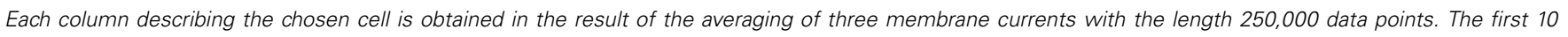
primary parameters are marked by a double line. The minimal and maximal values of each significant parameter in each row are bolded.

Table 2 | The collection of 20 significant parameters calculated for 6 files corresponding to currents recorded with the empty electrode placed inside an artificial cerebrospinal fluid (the biological material is absent).

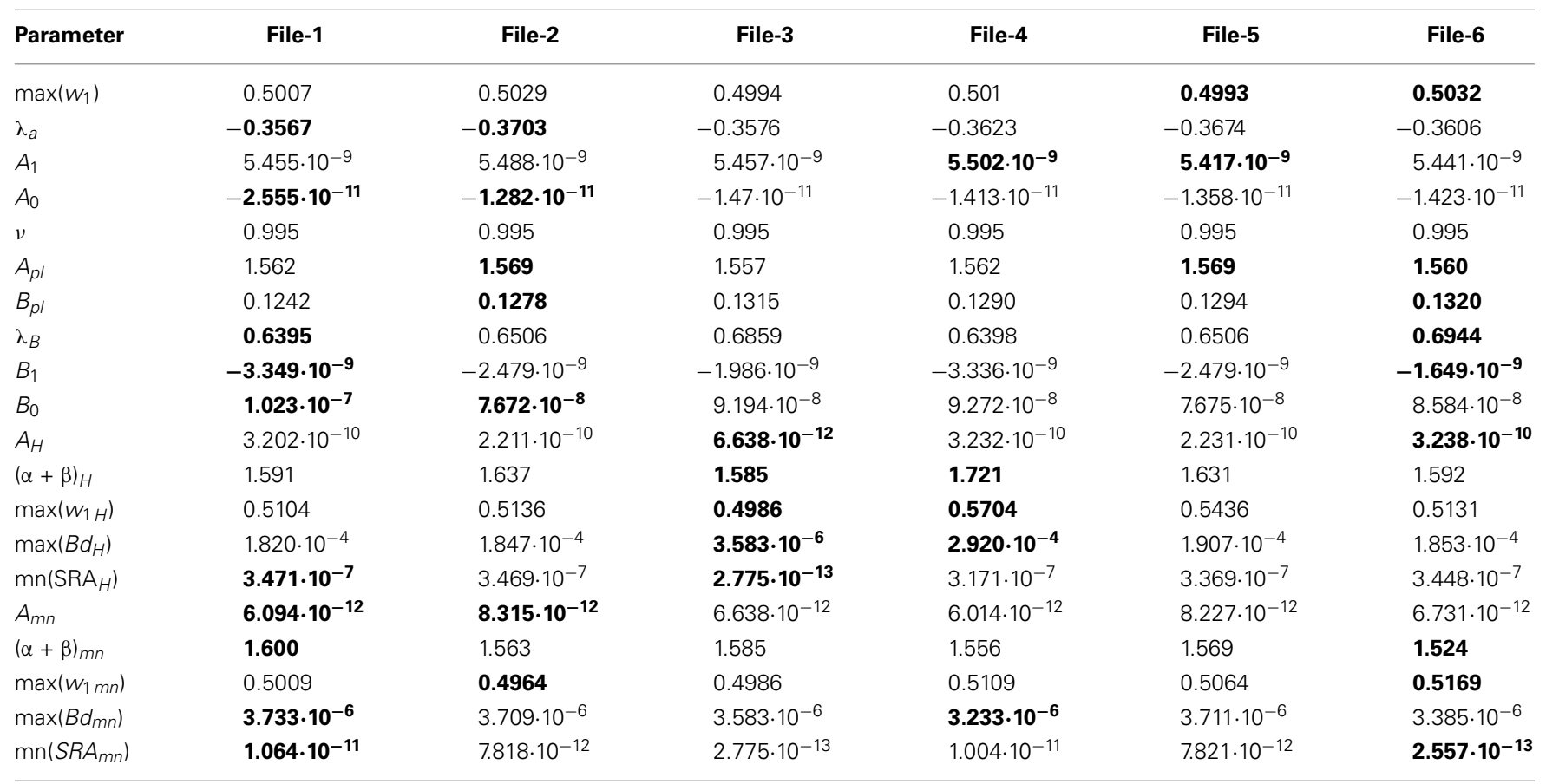

The first 10 primary parameters are marked by a double line. The minimal and maximal values of each significant parameter in each row are bolded. 
Table 3 | The correlation matrix of the calculated CC-factors [Expression (22)] for 20 parameters characterizing 7 neurons collected in the Table 1.

\begin{tabular}{|c|c|c|c|c|c|c|c|}
\hline Cells & Cell-1 & Cell-2 & Cell-3 & Cell-4 & Cell-5 & Cell-6 & Cell-7 \\
\hline Cell-1 & 1 & 0.99876 & 0.92838 & 0.99957 & 0.99841 & 0.9767 & 0.94824 \\
\hline Cell-2 & 0.99876 & 1 & 0.93615 & 0.99954 & 0.99981 & 0.98465 & 0.95698 \\
\hline Cell-3 & 0.92838 & 0.93615 & 1 & 0.93354 & 0.93708 & 0.97193 & 0.99714 \\
\hline Cell-4 & 0.99957 & 0.99954 & 0.93354 & 1 & 0.99933 & 0.98166 & 0.95451 \\
\hline Cell-5 & 0.99841 & 0.99981 & 0.93708 & 0.99933 & 1 & 0.98558 & 0.95776 \\
\hline Cell-6 & 0.9767 & 0.98465 & 0.97193 & 0.98166 & 0.98558 & 1 & 0.9804 \\
\hline Cell-7 & 0.94824 & 0.95698 & 0.99714 & 0.95451 & 0.95776 & 0.9804 & 1 \\
\hline
\end{tabular}

The maximal and minimal values of correlations in each row are bolded.

Table 4 | The correlation matrix of the calculated CC-factors for 20 parameters characterizing 6 empty electrode records collected in the Table 2.

\begin{tabular}{|c|c|c|c|c|c|c|}
\hline Files & $F-1$ & $F-2$ & $F-3$ & $F-4$ & F-5 & F-6 \\
\hline$F-2$ & 0.99581 & 1 & 0.97241 & 0.99767 & 0.99995 & 0.99951 \\
\hline$F-4$ & 0.99076 & 0.99767 & 0.97008 & 1 & 0.99706 & 0.99588 \\
\hline$F-5$ & 0.99642 & 0.99995 & 0.97244 & 0.99706 & 1 & 0.9996 \\
\hline
\end{tabular}

The maximal and minimal values of correlations in each row are bolded.

Table 5 | The correlation matrix of the CC-factors calculated for 7 cells and 6 empty electrodes.

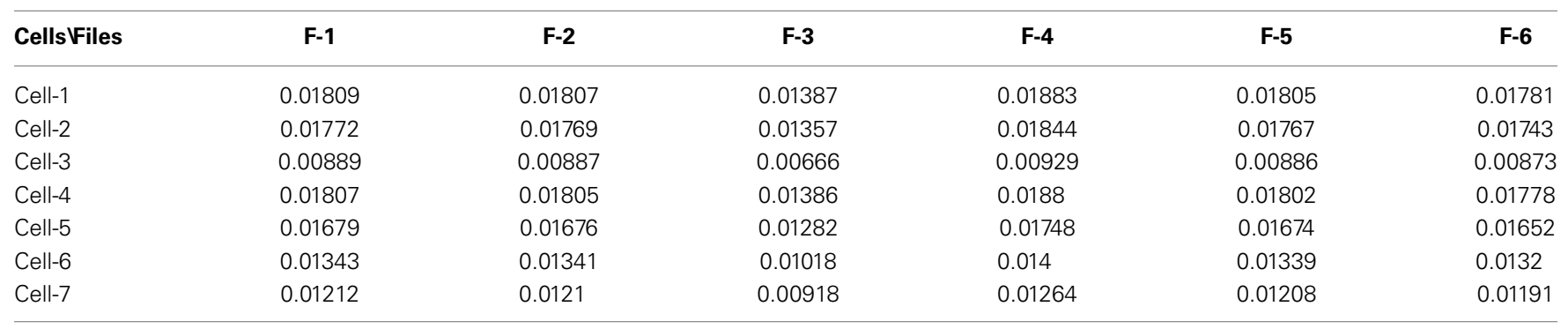

the membrane comprises a lot of elements which produce socalled "membrane noise"-rather small variations of membrane potential or trans-membrane current; mainly they are different types of ion channels, transporters and pumps. There are many active substances affecting the operation of these elements so the action of these substances actually can be detected by analyzing the membrane noise. But even if some substance does not affect channels, transporters or pumps directly its action often can be detected by noise analysis too. For example if the substance affects $G$ protein-coupled receptors or state of membrane lipids - in many cases it leads to the changes in the functioning of ion channels (Tillman and Cascio, 2003; Inanobe and Kurachi, 2014) and, accordingly, to the noise changes. So the analysis of the long-time series of noise can help to detect the action of many substances when we cannot detect this action differently.

For analysis of the long-time series we applied new BRC method based on the beta-distribution function. Four parameters of the beta-distribution function can be used for description of the local fluctuations and the averaged beta-distributions can be applied for quantitative reading of series containing large number of data points. The fluctuation spectroscopy based on beta distribution allows realizing the essential reduction $(2.5-10) \cdot 10^{5}$ data points to 20 quantitative parameters only [see Expressions (14)(16)] that contain the basic information calculated from three basic beta-distributions: (1) distribution over different segments (scales), (2) the secondary beta-distributions over their heights and (3) distributions over mean values. This reduction becomes possible thanks to the invariant properties that are expressed by formulae (3) and (5). We suppose that this approach can be applied successfully for the unified additional analysis of fluctuations of different long-time series that present the results of monitoring of biological, medical and other data reflecting the results of response of the complex system considered with respect to some external factor. In particular, this BRC method is applicable to testing the action of antagonist of receptor and ion channels when the modification based on different type of interaction (with binding site or with the open channel with different kinetics). In such experiments in order to understand the 
mechanism of action of some new substances we only need to compare the FSBD parameter changes caused by this substance with typical changes stored in the database.

\section{FUNDING}

This work was partially supported (Andrei I. Skorinkin) by RF grant "Leading Scientific School" and RFBR grant.

\section{ACKNOWLEDGMENTS}

We are grateful to professor Peter Illes (Leipzig University, Germany) for the possibility to receive the used experimental data in his laboratory, we also thank professor Sverre Holm (University of Oslo, Norway) for useful discussions. The work is performed according to the Russian Government Program of Competitive Growth of Kazan Federal University.

\section{REFERENCES}

Alvarez, O., Gonzalez, C., and Latorre, R. (2002). Counting channels: a tutorial guide on ion channel fluctuation analysis. Adv. Physiol. Educ. 26, 327-341. doi: 10.1152/advan.00006.2002

Baleanu, C. M., Nigmatullin, R. R., Cetin, S. S., Baleanu, D., and Ozcelik, S. (2011). New method and treatment technique applied to interband transition in $\mathrm{GaAs}_{1-x} \mathrm{P}_{x}$ ternary alloys. Cent. Eur. J. Phys. 9, 729-739. doi: 10.2478/s11534010-0068-y

Burr, R. L., Kirkness, C. J., and Mitchell, P. H. (2008). Detrended fluctuation analysis of the ICP predicts outcome following traumatic brain injury. IEEE Trans. Biomed. Eng. 55, 2509-2518. doi: 10.1109/TBME.2008.2001286

Ciurea, M. L., Lazanu, S., Stavaracher, I., Lepadatu, A.-M., Iancu, V., Mitroi, M. R., et al. (2011). Stress-induced traps in multilayered structures. J. Appl. Phys. 109, 013717. doi: 10.1063/1.3525582

Feder, J. (1988). Fractals. New York, NY: Plenum Press.

Gao, J. B., Hu, J., Tung, W. W., and Cao, Y. H. (2006). Distinguishing chaos from noise by scale-dependent Lyapunov exponent. Phys. Rev. E 74, 066204. doi: 10.1103/PhysRevE.74.066204

Gao, J. B., Sultan, H., Hu, J., and Tung, W. W. (2010). Denoising nonlinear time series by adaptive filtering and wavelet shrinkage: a comparison. IEEE Signal Proc. Let. 17, 237-240. doi: 10.1109/LSP.2009.2037773

Gao, J. B., Hu, J., and Tung, W. W. (2011). Facilitating joint chaos and fractal analysis of biosignals through nonlinear adaptive filtering. PLOS ONE 6:e24331. doi: 10.1371/journal.pone.0024331

Gao, J. B., Hu, J., Mao, X., and Perc, M. (2012a). Culturomics meets random fractal theory: insights into long-range correlations of social and natural phenomena over the past two centuries. J. R. Soc. Int. 9, 1956-1964. doi: 10.1098/rsif.2011.0846

Gao, J. B., Hu, J., Tung, W. W., and Blasch, E. (2012b). Multiscale analysis of physiological data by scale-dependent Lyapunov exponent. Front. Physiol. 2:110. doi: 10.3389/fphys.2011.00110

Gao, J. B., Gurbaxani, B. M., Hu, J., Heilman, K. J., Emanuele, V. A., Lewis, G. F., et al. (2013). Multiscale analysis of heart rate variability in nonstationary environments. Front. Physiol. 4:119. doi: 10.3389/fphys.2013.00119

Hausdorff, J. M., Peng, C.-K., Ladin, Z., Wei, J. Y., and Goldberger, A. L. (1995). Is walking a random walk? Evidence for long-range correlations in the stride interval of human gait. J. Appl. Physiol. 78, 349-358.

Hausdorff, J. M., Purdon, P., Peng, C.-K., Ladin, Z., Wei, J. Y., and Goldberger, A. L. (1996). Fractal dynamics of human gait: stability of long-range correlations in stride interval fluctuations. J. Appl. Physiol. 80, 1448-1457.

$\mathrm{Hu}$, J., Gao, J. B., Tung, W. W., and Cao, Y. H. (2010). Multiscale analysis of heart rate variability: a comparison of different complexity measures. Ann. Biomed. Eng. 38, 854-864. doi: 10.1007/s10439-009-9863-2

Inanobe, A., and Kurachi, Y. (2014). Membrane channels as integrators of G-protein-mediated signaling. Biochim. Biophys. Acta 1838, 521-531. doi: 10.1016/j.bbamem.2013.08.018

Jospin, M., Caminal, P., Jensen, E. W., Litvan, H., Vallverdu, M., Struys, R. F., et al. (2007). Detrended fluctuation analysis of EEG as a measure of depth of anesthesia. IEEE Trans. Biomed. Eng. 54, 840-846. doi: 10.1109/TBME.2007.893453
Kantelhardt, J. W., Koscielny-Bunde, E., Rego, H. H. A., Havlin, S., and Bunde, A. (2001). Detecting long-range correlations with detrended fluctuation analysis. Physica A 295, 441-454. doi: 10.1016/S0378-4371(01) 00144-3

Kuznetsov, N., Bonnette, S., Gao, J. B., and Riley, M. A. (2013). Adaptive fractal analysis reveals limits to fractal scaling in center of pressure trajectories. Ann. Biomed. Eng. 41, 1646-1660. doi: 10.1007/s10439-012-0646-9

Läuger, P. (1985). Structural fluctuations and current noise of ionic channels. Biophys. J. 48, 369-373. doi: 10.1016/S0006-3495(85)83793-0

Neher, E., and Sakmann, B. (1976). Noise analysis of drug induced voltage clamp currents in denervated frog muscle fibres. J. Physiol. 258, 705-729.

Nigmatullin, R. R. (2010). Universal distribution function for the stronglycorrelated fluctuations: general way for description of random sequences. Commun. Nonlinear Sci. Numer. Simulat. 15, 637-647. doi: 10.1016/j.cnsns. 2009.05.019

Nigmatullin, R. R., Ionescu, C., and Baleanu, D. (2012). NIMRAD: novel technique for respiratory data treatment. J. Signal Image Video Process. doi: 10.1007/ s11760-012-0386-1

Ossadnik, S. M., Buldyrev, S. V., Goldberger, A. L., Havlin, S., Mantegna, R. N., Peng, C.-K., et al. (1994). Correlation approach to identify coding regions in DNA sequences. Biophys. J. 67, 64-70. doi: 10.1016/S0006-3495(94) 80455-2

Peng, C.-K., Buldyrev, S. V., Havlin, S., Simons, M., Stanley, H. E., and Goldberger, A. L. (1994). Mosaic organization of DNA nucleotides. Phys. Rev. E 49, 1685-1689. doi: 10.1103/PhysRevE.49.1685

Peng, C.-K., Havlin, S., Stanley, H. E., and Goldberger, A. L. (1995). Quantification of scaling exponents and crossover phenomena in nonstationary heartbeat time series. Chaos 5, 82-87. doi: 10.1063/1.166141

Penzel, T., Kantelhardt, J. W., Grote, L., Peter, J.-H., and Bunder, A. (2003) Comparison of deterended fluctuation analysis and spectral analysis for heart rate variability in sleep and sleep apnea. IEEE Trans. Biomed. Eng. 50, 1143-1151. doi: 10.1109/TBME.2003.817636

Riley, M. A., Kuznetsov, N., Bonnette, S., Wallot, S., and Gao, J. B. (2012). A tutorial introduction to adaptive fractal analysis. Front. Physiol. 3, 1-10. doi: 10.3389/fphys.2012.00371

Sigworth, F. J. (1980). The variance of sodium current fluctuations at the node of Ranvier. J. Gen. Physiol. 307, 97-129.

Sigworth, F. J. (1985). Open channel noise. I. Noise in acetylcholine receptor currents suggests conformational fluctuations. Biophys. J. 47, 709-720. doi: 10.1016/S0006-3495(85)83968-0

Sigworth, F. J. (1986). Open channel noise. II. A test for coupling between current fluctuations and conformational transitions in the acetylcholine receptor. Biophys. J. 49, 1041-1046. doi: 10.1016/S0006-3495(86)83732-8

Tillman, T. S., and Cascio, M. (2003). Effects of membrane lipids on ion channel structure and function. Cell Biochem. Biophys. 38, 161-190. doi: 10.1385/CBB:38:2:161

Traynelisa, S. F., and Jaramilloa, F. (1998). Getting the most out of noise in the central nervous system. Trends Neurosci. 21, 137-145. doi: 10.1016/S01662236(98)01238-7

Venkataramanan, L., and Sigworth, F. J. (2002). Applying hidden Markov models to the analysis of single ion channel activity. Biophys. J. 82, 1930-1942. doi: 10.1016/S0006-3495(02)75542-2

Conflict of Interest Statement: The authors declare that the research was conducted in the absence of any commercial or financial relationships that could be construed as a potential conflict of interest.

Received: 04 June 2014; paper pending published: 11 July 2014; accepted: 08 September 2014; published online: 26 September 2014.

Citation: Nigmatullin RR, Giniatullin RA and Skorinkin AI (2014) Membrane current series monitoring: essential reduction of data points to finite number of stable parameters. Front. Comput. Neurosci. 8:120. doi: 10.3389/fncom.2014.00120 This article was submitted to the journal Frontiers in Computational Neuroscience. Copyright (c) 2014 Nigmatullin, Giniatullin and Skorinkin. This is an open-access article distributed under the terms of the Creative Commons Attribution License (CC BY). The use, distribution or reproduction in other forums is permitted, provided the original author(s) or licensor are credited and that the original publication in this journal is cited, in accordance with accepted academic practice. No use, distribution or reproduction is permitted which does not comply with these terms. 